\title{
Derived Categories and Birational Geometry
}

\author{
Yujiro Kawamata
}

October 22, 2018

\section{Introduction}

This paper is concerned with a surprizing parallelism between minimal model program and semi-orthogonal decompositions of derived categories found by Bondal and Orlov ([3]).

The bounded derived category of coherent sheaves on a smooth projective variety has a finiteness property called saturatedness (§2). A map between smooth projective varieties such as a projective space bundle, a blowing up along a smooth center or a standard smooth flip induces a semi-orthogonal decomposition of the derived category (§3). We expect that one can attach a saturated triangulated category to any singular variety which appears in the minimal model program, and that a map between these varieties such as a Mori fiber space, a divisorial contraction or a flip induces a semi-orthogonal decomposition of the category.

We review some basic results on categories in $\S 2$ following [2]. We treat some cases which motivate our expectation in $\S 3$. The cases include smooth varieties due to [13] and [3], and toric varieties [9]. We provide some properties of the desired category, and give a possible definition of the correct category for certain three dimensional cases by calculating the behavior of the category under the divisorial contractions of smooth threefolds in $\S 4$. We conclude this paper with a short section of open questions.

The author would like to thank the referee for a careful reading and useful suggestions. 


\section{Saturated categories and semi-orthogonal decompositions}

Let $X$ be a projective variety over a field $k$. The largest derived category for $X$ is the unbounded derived category of quasi-coherent sheaves denoted by $D(\mathrm{QCoh}(X))$ (we refer to [6] for basic definitions). There are functors such as $f_{*}, f^{*}, f^{!}$for morphisms $f: X \rightarrow Y$, and the Grothendieck duality theorem holds $([12])$. This category is $k$-linear in the sense that the set of morphisms $\operatorname{Hom}(a, b)$ for $a, b \in D(\mathrm{QCoh}(X))$ has the structure of a $k$-vector space. It is, however, infinite dimensional and we might hope to work with categories for which the Hom space has finite dimension.

A candidate is the bounded derived category of coherent sheaves denoted by $D^{b}(\operatorname{Coh}(X))$. If $X$ is smooth, then $D^{b}(\operatorname{Coh}(X))$ is of finite type in the

sense that $\sum_{p \in \mathbb{Z}} \operatorname{dim} \operatorname{Hom}(a, b[p])$ is finite. But if $X$ is singular, then the homological dimension is infinite. Indeed, if $x \in X$ is a singular point, then there are infinitely many $p$ such that $\operatorname{Hom}\left(\mathcal{O}_{x}, \mathcal{O}_{x}[p]\right) \neq 0$, where $\mathcal{O}_{x}$ denotes a skyscraper sheaf of length 1 at $x$.

An object $c$ in a triangulated category $D$ having arbitrary coproducts is said to be compact if $\operatorname{Hom}\left(c, \coprod_{\lambda} a_{\lambda}\right) \cong \coprod_{\lambda} \operatorname{Hom}\left(c, a_{\lambda}\right)$. An object $c \in$ $D(\mathrm{Q} C o h(X))$ is compact if and only if it is perfect in the sense that it is locally isomorphic to a bounded complex of locally free coherent sheaves. Let $\operatorname{Perf}(X)=D(\mathrm{QCoh}(X))^{c}$ be the triangulated category of perfect complexes. It is a full subcategory of $D^{b}(\operatorname{Coh}(X))$, and they coincide if $X$ is smooth.

If $a \in \operatorname{Perf}(X)$ and $b \in D^{b}(\operatorname{Coh}(X))$, then $\sum_{p \in \mathbb{Z}} \operatorname{dim} \operatorname{Hom}(a, b[p])<\infty$. Serre duality holds

$$
\operatorname{Hom}(a, b) \cong \operatorname{Hom}\left(b, a \otimes \omega_{X}^{\bullet}\right)^{*}
$$

for $a \in \operatorname{Perf}(X)$ and $b \in D^{b}(\operatorname{Coh}(X))$, where $\omega_{X}^{\bullet}$ is a dualizing complex. For example, if $X$ is smooth, then $\omega_{X}^{\bullet}=\omega_{X}[\operatorname{dim} X]$. Therefore, $\operatorname{Perf}(X)$ is similar to homology while $D^{b}(\operatorname{Coh}(X))$ cohomology, because Serre duality is similar to Poincaré duality. In particular, if $X$ is smooth, then the functor $S_{X}: D^{b}(\operatorname{Coh}(X)) \rightarrow D^{b}(\operatorname{Coh}(X))$ defined by $S_{X}(a)=a \otimes \omega_{X}[\operatorname{dim} X]$ is a Serre functor in the sense that

$$
\operatorname{Hom}(a, b) \cong \operatorname{Hom}\left(b, S_{X}(a)\right)^{*}
$$

for $a, b \in D^{b}(\operatorname{Coh}(X))$. 
It is important to consider singular varieties in minimal model theory. Since the sets $\operatorname{Hom}\left(\mathcal{O}_{x}, \mathcal{O}_{x}[p]\right)$ do not vanish for $p \geq 0$ but vanish for $p<0$ for a singular point $x \in X$, there cannot be a Serre functor in the category $D^{b}(\operatorname{Coh}(X))$ for a singular variety $X$. So we would like to have something like intersection homology for a singular variety $X$ that lies between $\operatorname{Perf}(X)$ and $D^{b}(\operatorname{Coh}(X))$ and has a Serre functor.

A $k$-linear triangulated category $D$ of finite type is said to be saturated if any cohomological functor $F: D^{\mathrm{op}} \rightarrow$ ( $k$-vect) of finite type is representable. That is, if $\sum_{p \in \mathbb{Z}} \operatorname{dim} F(a[p])<\infty$ for any $a \in D$, then there exists $b \in D$ such that $F(a) \cong \operatorname{Hom}(a, b)$. A saturated category has always a Serre functor, because the dual of the left hand side of (2.1) defines a cohomological functor. The category $D^{b}(\operatorname{Coh}(X))$ for smooth $X$ is saturated (4)). For example, an object $a \in D$ in a $k$-linear triangulated category is said to be exceptional if $\operatorname{Hom}(a, a[p])=0$ for $p \neq 0$ while $\operatorname{Hom}(a, a) \cong k$. In this case, the subcategory $\langle a\rangle$ generated by $a$ is saturated because it is equivalent to $D^{b}(\operatorname{Coh}(\operatorname{Spec} k))$.

A triangulated full subcategory $B$ of a triangulated category $A$ is said to be right admissible if the embedding $j_{*}: B \rightarrow A$ has a right adjoint functor $j^{!}: A \rightarrow B$. Let $C=B^{\perp}$ be the right orthogonal defined by

$$
C=\{c \in A \mid \operatorname{Hom}(b, c)=0 \forall b \in B\} .
$$

Then an arbitrary $a \in A$ has a unique presentation by a distinguished triangle

$$
b \rightarrow a \rightarrow c \rightarrow b[1]
$$

for $b=j_{*} j^{!} a \in B$ and $c \in C$. We write this as

$$
A=\langle C, B\rangle
$$

and call it a semi-orthogonal decomposition of $A$. We note that the form $\operatorname{Hom}(a, b)$ is similar to a bilinear form on $A$, but it is not symmetric. Thus the orthogonality is only one-sided. Indeed, $D^{b}(\operatorname{Coh}(X))$ is indecomposable with respect to Hom as long as $X$ is irreducible ([5]). We denote by $\langle D, C, B\rangle$ for $\langle D,\langle C, B\rangle\rangle$, etc. For example, if $A$ is of finite type and $B$ is saturated, then $B$ is always right admissible. Conversely, if $A$ is saturated and $B$ is right admissible, then $B$ is saturated.

If $A$ has a Serre functor $S_{A}$, then $B$ has also a Serre functor given by $S_{B}=j^{!} S_{A} j_{*}$, because

$$
\begin{aligned}
& \operatorname{Hom}_{B}(a, b) \cong \operatorname{Hom}_{A}\left(j_{*} a, j_{*} b\right) \\
& \cong \operatorname{Hom}_{A}\left(j_{*} b, S_{A} j_{*} a\right)^{*} \cong \operatorname{Hom}_{B}\left(b, j^{!} S_{A} j_{*} a\right)^{*}
\end{aligned}
$$


for $a, b \in B . j_{*}$ has also a left adjoint $j^{*}$ defined by $j^{*}=S_{B}^{-1} j^{!} S_{A}$, because

$$
\begin{aligned}
& \operatorname{Hom}_{A}\left(a, j_{*} b\right) \cong \operatorname{Hom}_{A}\left(j_{*} b, S_{A} a\right)^{*} \\
& \cong \operatorname{Hom}_{B}\left(b, j ! S_{A} a\right)^{*} \cong \operatorname{Hom}_{B}\left(j ! S_{A} a, S_{B} b\right)
\end{aligned}
$$

for $a \in A$ and $b \in B$.

Lemma 2.1. Assume that $X$ is singular. Then $\operatorname{Perf}(X)$ is not saturated.

Proof. Assume the contrary and let $x \in X$ be a singular point. Assume that the contravariant functor $\operatorname{Hom}\left(\bullet, \mathcal{O}_{x}\right)$ on $\operatorname{Perf}(X)$ is represented by an object $c \in \operatorname{Perf}(X)$ :

$$
\operatorname{Hom}\left(\bullet, \mathcal{O}_{x}\right) \cong \operatorname{Hom}(\bullet, c) .
$$

Let $x^{\prime} \neq x$ be another point and $b$ a coherent sheaf supported at $x^{\prime}$ such that $b \in \operatorname{Perf}(X)$ as an object in $D^{b}(\operatorname{Coh}(X))$. Then we have

$$
\operatorname{Hom}(b, c) \cong \operatorname{Hom}\left(b, \mathcal{O}_{x}\right)=0 .
$$

Hence $\operatorname{Supp}(c)=\{x\}$. Since

$$
\operatorname{Hom}\left(\mathcal{O}_{X}, c\right) \cong \operatorname{Hom}\left(\mathcal{O}_{X}, \mathcal{O}_{x}\right) \cong k
$$

we conclude that $c$ is a sheaf of length 1 supported at $x$, but the latter is not contained in $\operatorname{Perf}(X)$.

\section{Minimal model program and decomposi- tions}

The minimal model program consists of three kinds of basic operations, namely, Mori fiber spaces, divisorial contractions and flips (see for example [10]). The latter two are birational maps which decrease the canonical divisor $K$. The following are the simplest examples of these operations for smooth projective varieties. The point is that semi-orthogonal decompositions of the derived categories are parallel to the decompositions of canonical divisors.

Example 3.1. In this example, we consider only smooth projective varieties $X, Y$, etc. For simplicity, we write $D(X)$ for the bounded derived category of coherent sheaves $D^{b}(\operatorname{Coh}(X))$, etc. 
(1) ([13]) Let $f: X \rightarrow Y$ be a projective space bundle associated to a vector bundle of rank $r$. This is a Mori fiber space. The functor $f^{*}: D(Y) \rightarrow$ $D(X)$ is fully faithful, and there is a semi-orthogonal decomposition

$$
D(X)=\left\langle D(Y)_{-r+1}, \ldots, D(Y)_{-1}, D(Y)_{0}\right\rangle
$$

where the $D(Y)_{i}$ denote the subcategories $f^{*} D(Y) \otimes \mathcal{O}_{X}(i)$ of $D(X)$ for the tautological line bundle $\mathcal{O}_{X}(1)$.

(2) (13]) Let $C$ be a smooth subvariety of $Y$ of codimension $r \geq 2$ and $f: X \rightarrow Y$ the blowing up along $C$. This is a divisorial contraction. The exceptional divisor $E$ is a projective space bundle over $C$ as in (1). Let $f_{E}: E \rightarrow C$ be the induced morphism and $j: E \rightarrow X$ the embedding. Then the functors $f^{*}: D(Y) \rightarrow D(X)$ and $j_{*} f_{E}^{*}: D(C) \rightarrow D(X)$ are fully faithful, and there is a semi-orthogonal decomposition

$$
D(X)=\left\langle j_{*} f_{E}^{*} D(C)_{-r+1}, \ldots, j_{*} f_{E}^{*} D(C)_{-1}, f^{*} D(Y)\right\rangle
$$

where $j_{*} f_{E}^{*} D(C)_{i}=j_{*} f_{E}^{*} D(C) \otimes \mathcal{O}_{X}(-i E)$. We have a corresponding equality of canonical divisors

$$
K_{X}=f^{*} K_{Y}+(r-1) E .
$$

(3) ([3]) Let $E$ be a subvariety of $X$ which is isomorphic to a projective space $\mathbb{P}^{r-1}$ and such that the normal bundle $N_{E / X}$ is isomorphic to $\mathcal{O}_{\mathbb{P}^{r-1}}(-1)^{s}$. Let $f: Y \rightarrow X$ be the blowing up along $E$. Then there is a blowing down $f^{+}: Y \rightarrow X^{+}$of the exceptional divisor $F=f^{-1}(E)$ to another direction such that $E^{+}=f^{+}(F)$ is isomorphic to $\mathbb{P}^{s-1}$ and such that the normal bundle $N_{E^{+} / X^{+}}$is isomorphic to $\mathcal{O}_{\mathbb{P}^{s-1}}(-1)^{r}$. If $r>s$, then this is a flip, while if $r=s$, then it is a flop. If $r \geq s$, then the functor $f_{*} f^{+*}: D\left(X^{+}\right) \rightarrow D(X)$ is fully faithful, and there is a semi-orthogonal decomposition

$$
D(X)=\left\langle\mathcal{O}_{E}(s-r), \ldots, \mathcal{O}_{E}(-1), f_{*} f^{+*} D\left(X^{+}\right)\right\rangle
$$

where the subcategories $\left\langle\mathcal{O}_{E}(i)\right\rangle$ generated by the sheaves $\mathcal{O}_{E}(i)$ are denoted by $\mathcal{O}_{E}(i)$ for simplicity. In particular, we have an equivalence of triangulated categories $D(X) \cong D\left(X^{+}\right)$for the flop case. We have a corresponding equality of canonical divisors

$$
f^{*} K_{X}=f^{+*} K_{X^{+}}+(r-s) F \text {. }
$$


It is important to deal with singular varieties in the minimal model program. Therefore, we have to define good derived categories for such varieties. The simplest case is the one with quotient singularities. For a variety $X$ with only quotient singularities, we can naturally associate a smooth DeligneMumford stack $\mathcal{X}$. The set of points of the stack $\mathcal{X}$ is the same as that of the variety $X$, but the points on $\mathcal{X}$ have automorphism groups corresponding to the stabilizer groups of the points on $X$. The sheaves on $\mathcal{X}$ have actions by these groups. Let $D^{b}(\operatorname{Coh}(\mathcal{X}))$ be the bounded derived category of coherent sheaves on $\mathcal{X}$. For example, if $X=M / G$ is the quotient of a smooth variety $M$ by a finite group $G$, then $D^{b}(\operatorname{Coh}(\mathcal{X}))=D^{b}\left(\operatorname{Coh}^{G}(M)\right)$ is the bounded derived category of $G$-equivariant coherent sheaves on $M$. The following example suggests that the above $D^{b}(\operatorname{Coh}(\mathcal{X}))$ is the correct answer to our problem for varieties with only quotient singularities (cf. [8] and [9] for more justifications).

Example 3.2. ([7]) Let $X$ be a 4 dimensional smooth projective variety, $E$ a subvariety which is isomorphic to a projective plane $\mathbb{P}^{2}$ and such that the normal bundle $N_{E / X}$ is isomorphic to $\mathcal{O}_{\mathbb{P}^{2}}(-1) \oplus \mathcal{O}_{\mathbb{P}^{2}}(-2)$. Let $f_{1}$ : $X_{1} \rightarrow X$ be the blowing up along $E$. Then the exceptional divisor $F=$ $f_{1}^{-1}(E)$ contains a subvariety $E_{1}$ which is isomorphic to $\mathbb{P}^{2}$ and the normal bundle $N_{E_{1} / X_{1}}$ is isomorphic to $\mathcal{O}_{\mathbb{P}^{2}}(-1)^{2}$. Let $f_{2}: Y \rightarrow X_{1}$ be the further blowing up along $E_{1}$. Then there is a blowing down $f_{1}^{+}: Y \rightarrow X_{1}^{+}$of the exceptional divisor $F_{1}=f_{2}^{-1}\left(E_{1}\right)$ to another direction such that $E_{1}^{+}=f_{1}^{+}\left(F_{1}\right)$ is isomorphic to $\mathbb{P}^{1}$. The strict transform $F^{\prime}$ of $F$ on $X_{1}^{+}$is isomorphic to $\mathbb{P}^{2}$ and the normal bundle $N_{F^{\prime} / X_{1}^{+}}$is isomorphic to $\mathcal{O}_{\mathbb{P}^{2}}(-2)$. Let $f_{2}^{+}: X_{1}^{+} \rightarrow X^{+}$ be the blowing down of $F^{\prime}$. The image $Q=f_{2}^{+}\left(F^{\prime}\right)$ is an isolated quotient singularity with stabilizer group $\mathbb{Z} / 2$.

The composition $f_{2}^{+} f_{1}^{+} f_{2}^{-1} f_{1}^{-1}$ is a flop. Namely, we have an equality $f_{2}^{*} f_{1}^{*} K_{X}=f_{1}^{+*} f_{2}^{+*} K_{X^{+}}$. Correspondingly, we have an equivalence of derived categories

$$
f_{2 *}^{+} f_{1 *}^{+} f_{2}^{*} f_{1}^{*}: D^{b}(\operatorname{Coh}(X)) \cong D^{b}\left(\operatorname{Coh}\left(\mathcal{X}^{+}\right)\right)
$$

where we consider the associated stack $\mathcal{X}^{+}$instead of the underlying variety with a quotient singularity $X^{+}$. But the functor $\pi_{*}: D^{b}\left(\operatorname{Coh}\left(\mathcal{X}^{+}\right)\right) \rightarrow$ $D^{b}\left(\mathrm{Coh}\left(X^{+}\right)\right)$induced by the projection $\pi: \mathcal{X}^{+} \rightarrow X^{+}$is not an equivalence. For example,

$$
f_{2 *}^{+} f_{1 *}^{+} f_{2}^{*} f_{1}^{*}\left(\Omega_{E}^{1}(-1)\right)=\mathcal{O}_{Q}(1)
$$

and $\pi_{*} \mathcal{O}_{Q}(1) \cong 0$, where $\mathcal{O}_{Q}(1)$ is a skyscraper sheaf of length 1 supported at $Q$ on which the stabilizer group acts non-trivially. Thus an ordinary sheaf 
$\Omega_{E}^{1}(-1)$ on $X$ corresponds to an equivariant sheaf $\mathcal{O}_{Q}(1)$ on the imaginary stack $\mathcal{X}^{+}$which disappears on the real variety $X^{+}$.

The following example shows that the semi-orthogonal decompositions of derived categories are governed by the inequalities of canonical divisors and not by the directions of morphisms. This fact suggests a distinguished status of the canonical divisors in the theory of derived categories. We note that the derived categories contain almost all information on varieties in a similar way like the motives (cf. [14]).

Example 3.3. (9]) Let $X$ be a smooth projective variety of dimension $n$ which contains a divisor $E$ being isomorphic to a projective space $\mathbb{P}^{n-1}$ and such that the normal bundle is isomorphic to $\mathcal{O}_{\mathbb{P}^{n-1}}(-k)$ for an integer $k>0$. Let $f: X \rightarrow Y$ be the blowing down of $E$. Then $Y$ has an isolated quotient singularity $Q$ whose stabilizer group is isomorphic to $\mathbb{Z} / k$. Let $\mathcal{Y}$ be the associated smooth Deligne-Mumford stack, and let $\mathcal{Z}=X \times_{Y} \mathcal{Y}$ be the fiber product with projections $\pi: \mathcal{Z} \rightarrow X$ and $\tilde{f}: \mathcal{Z} \rightarrow \mathcal{Y}$. We have an equality

$$
K_{X}=f^{*} K_{Y}+\frac{n-k}{k} E .
$$

If $n>k$, then we have $K_{X}>f^{*} K_{Y}$. Correspondingly, the functor $\pi_{*} \tilde{f}^{*}$ : $D^{b}(\operatorname{Coh}(\mathcal{Y})) \rightarrow D^{b}(\operatorname{Coh}(X))$ is fully faithful, and there is a semi-orthogonal decomposition

$$
D^{b}(\operatorname{Coh}(X))=\left\langle\mathcal{O}_{E}(-n+k), \ldots, \mathcal{O}_{E}(-1), \pi_{*} \tilde{f}^{*} D^{b}(\operatorname{Coh}(\mathcal{Y}))\right\rangle .
$$

On the other hand, if $n<k$, then we have $K_{X}<f^{*} K_{Y}$. Correspondingly, the functor $\tilde{f}_{*} \pi^{*}: D^{b}(\operatorname{Coh}(X)) \rightarrow D^{b}(\operatorname{Coh}(\mathcal{Y}))$ is fully faithful, and there is a semi-orthogonal decomposition

$$
D^{b}(\operatorname{Coh}(\mathcal{Y}))=\left\langle\mathcal{O}_{Q}(-n), \ldots, \mathcal{O}_{Q}(-k+1), \tilde{f}_{*} \pi^{*} D^{b}(\operatorname{Coh}(X))\right\rangle
$$

where $\mathcal{O}_{Q}(i)$ denotes a skyscraper sheaf of length 1 at $Q$ with a suitable action by the stabilizer group. In particular, if $n=k$, then there is an equivalence $\pi_{*} \tilde{f}^{*}: D^{b}(\operatorname{Coh}(\mathcal{Y})) \cong D^{b}(\operatorname{Coh}(X))$.

We note that the functors are given by the pull-backs and the push-downs as in the case of flips. Indeed, divisorial contractions and flips are very similar operations from the view point of the minimal model program.

The above picture extends for $\mathbb{Q}$-factorial toric varieties $([9])$ : 
Theorem 3.4. Let $f: X-\rightarrow Y$ be a toric divisorial contraction or flip between $\mathbb{Q}$-factorial projective toric varieties, and let $\mathcal{X}$ and $\mathcal{Y}$ be their associated smooth Deligne-Mumford stacks. Then there is a semi-orthogonal decomposition

$$
D^{b}(\operatorname{Coh}(\mathcal{X})) \cong\left\langle C, D^{b}(\operatorname{Coh}(\mathcal{Y}))\right\rangle
$$

which is described in detail in terms of toric fans for $X$ and $Y$.

We note that $\mathbb{Q}$-factorial toric varieties have only quotient singularities. We have similar results for toric Mori fiber spaces. The theorem has a log version in the case where the coefficients of the boundary are of the form $1-\frac{1}{m}$ for some positive integers $m$. In particular, toric flops induce derived equivalences. We refer to [9] for details. As a corollary, we obtain the McKay correspondence for abelian quotient singularities:

Corollary 3.5. Let $X$ be a projective variety with only quotient singularities whose stabilizer groups are abelian groups whose orders are prime to the characteristic of the base field, and let $f: Y \rightarrow X$ be a projective crepant resolution, i.e., $Y$ is smooth, $f$ is projective and birational, and $K_{Y}=f^{*} K_{X}$. Then there is an equivalence of triangulated categories

$$
D^{b}(\operatorname{Coh}(Y)) \cong D^{b}(\operatorname{Coh}(\mathcal{X}))
$$

Proof. We may replace $X$ by its local model and assume that $X$ is toric by [5]. Then there exists a toric crepant $\mathbb{Q}$-factorial terminalization $f^{\prime}: Y^{\prime} \rightarrow X$. Let $H$ be an $f$-ample divisor on $Y$ and $H^{\prime}$ its strict transform on $Y^{\prime}$. Since $Y^{\prime}$ is toric, $H^{\prime}$ is linearly equivalent to a toric divisor which is denoted by $H^{\prime}$ again. We proceed by MMP with respect to $\left(Y^{\prime}, \epsilon H^{\prime}\right)$ over $X$ for a small positive number $\epsilon$. Since the pair is toric, the process is a toric MMP. After finitely many steps, we reach a log minimal model that is isomorphic to $Y$. Therefore, $f: Y \rightarrow X$ is also toric. The MMP over $X$ starting from $(Y, B)$ for a suitable toric boundary $B$ ends at $X$. Therefore, $Y$ and the smooth Deligne-Mumford stack over $X$ are derived equivalent by the theorem (see also [8]).

There is a warning. The derived category may have semi-orthogonal decompositions beyond the minimal model program. For example, the derived category of a projective space has a complete semi-orthogonal decomposition to exceptional objects by [1]. This fact is extended to an arbitrary $\mathbb{Q}$-factorial projective toric variety $([9])$. The derived categories of some Fano manifolds 
have interesting semi-orthogonal decompositions which reflect the geometry of these manifolds ([1] ). Even minimal varieties such as Enriques surfaces have derived semi-orthogonal decompositions. Therefore, MMP is only a preparation as in the case of the classification of surfaces. After that, deeper decompositions may be possible like in the case of decompositions of motives.

\section{Divisorial contractions of smooth 3-folds}

We would like to define a correct category for an arbitrary variety which appears in the minimal model program, or in the Mori category. Thus let $X$ be a projective variety with only terminal singularities, and $f: Y \rightarrow X$ a resolution of singularities. As a working hypothesis, we look for a minimal saturated subcategory $D=D(X)$ of $D^{b}(\operatorname{Coh}(Y))$ which contains $f^{*} \operatorname{Perf}(X)$ :

$$
f^{*} \operatorname{Perf}(X) \subset D(X) \subset D^{b}(\operatorname{Coh}(Y))
$$

if it exists and unique up to equivalence. If $X$ is smooth, then we have $D(X)=D^{b}(\operatorname{Coh}(X))$.

As a first step, we consider a divisorial contraction $f: Y \rightarrow X$ of a smooth 3 dimensional variety to a singular variety. The morphism $f$ is an isomorphism outside a prime divisor $E$ on $Y$, and $P=f(E)$ is the singular point of $X$. There are three cases, where two of them has already answers described in $\S 3$. The above working hypothesis seems to produce the same categories in these cases.

\subsection{Case 1}

$E$ is isomorphic to a smooth quadric surface $\mathbb{P}^{1} \times \mathbb{P}^{1}$ with normal bundle $\mathcal{O}_{E}(-1,-1)$, where the pair of rulings on $E$ are numerically equivalent to each other on $X$.

Let

$$
C=\left\langle\mathcal{O}_{E}(-1,-1), \mathcal{O}_{E}(0,-1)\right\rangle \subset D^{b}(\operatorname{Coh}(Y))
$$

be the subcategory generated by a sequence of exceptional objects, and let $D={ }^{\perp} C$. Since $C$ is equivalent to $D^{b}\left(\operatorname{Coh}\left(\mathbb{P}^{1}\right)\right)$, it is admissible, and $D$ is saturated. Since $f_{*} c=0$ for any $c \in C$, we have $f^{*} p \in D$ for $p \in \operatorname{Perf}(X)$, thus $f^{*} \operatorname{Perf}(X) \subset D$.

If $k=\mathbb{C}$, then there are two small resolutions $g_{i}: Y_{i} \rightarrow X(i=1,2)$ in the analytic category, with analytic divisorial contractions $f_{i}: Y \rightarrow Y_{i}$ such that 
$f_{i}(E)$ is isomorphic to $\mathbb{P}^{1}$. We can check that $D=f_{1}^{*} D^{b}\left(\operatorname{Coh}\left(Y_{1}\right)\right)$, and the latter should be the correct category since $Y_{1}$ is smooth. Since $g_{1}$ is crepant, it follows that $S_{D}(d) \cong d[3]$ if $d \in D$ and $f_{*} d=0$. We can also prove this fact for general $k$, because these objects are concentrated on the divisor $E$ and the global structure of $X$ is irrelevant.

Lemma 4.1. $D$ is minimal in the sense that $D$ has no semi-orthogonal decomposition relative to $X$, i.e., a semi-orthogonal decomposition such that one of the factors contains $f^{*} \operatorname{Perf}(X)$.

Proof. Suppose there is still a semi-orthgonal decomposition $D=\left\langle C^{\prime}, D^{\prime}\right\rangle$ such that $f^{*} \operatorname{Perf}(X) \subset D^{\prime}$. Since $C^{\prime} \subset\left(f^{*} \operatorname{Perf}(X)\right)^{\perp}$, we have $f_{*} c=0$ for $c \in C^{\prime}$. Then we have for $c \in C^{\prime}$ and $d \in D^{\prime}$

$$
\operatorname{Hom}(c, d) \cong \operatorname{Hom}(d, c[3])^{*} \cong 0 .
$$

Hence $D$ is decomposable; $D=C^{\prime} \oplus D^{\prime}$.

Assuming that $k=\mathbb{C}$, let $a_{y}=f_{1}^{*} \mathcal{O}_{y} \in D$ for a point $y \in Y_{1}$. Since $\operatorname{Hom}\left(a_{y}, a_{y}\right) \cong k$, we have either $a_{y} \in C^{\prime}$ or $a_{y} \in D^{\prime}$. Since $f_{*} a_{y} \neq 0$, we conclude that $a_{y} \in D^{\prime}$. But let $c^{\prime} \in C^{\prime}$ be a non-zero object, and write $c^{\prime}=$ $f_{1}^{*} c$. If we take a point $y$ in the support of $c$, then we have $\operatorname{Hom}\left(c, \mathcal{O}_{y}[p]\right) \neq 0$ for some $p$, hence $\operatorname{Hom}\left(c^{\prime}, a_{y}[p]\right) \neq 0$. But this is a contradiction. For general $k$, we can still define $a_{y}$ because it is supported on $E$, and the above argument works.

If we take

$$
C_{1}=\left\langle\mathcal{O}_{E}(-1,-1), \mathcal{O}_{E}(-1,0)\right\rangle \subset D^{b}(\operatorname{Coh}(Y))
$$

instead of $C$ and define $D_{1}={ }^{\perp} C_{1}$, then $D$ and $D_{1}$ are equivalent, because $Y_{1}$ and $Y_{2}$ are related by a standard flop.

There is another candidate for an admissible subcategory which is more symmetric than $C$ or $C_{1}$. Let

$$
\tilde{C}=\left\langle\mathcal{O}_{E}(-1,0), \mathcal{O}_{E}(0,-1)\right\rangle \subset D^{b}(\operatorname{Coh}(Y))
$$

and let $\tilde{D}={ }^{\perp} \tilde{C}$ be the right orthogonal. We have again $f^{*} \operatorname{Perf}(X) \subset \tilde{D}$. The generators of $\tilde{C}$ are exceptional objects,

$$
\operatorname{Hom}\left(\mathcal{O}_{E}(-1,0), \mathcal{O}_{E}(0,-1)[2]\right) \cong \operatorname{Hom}\left(\mathcal{O}_{E}(0,-1), \mathcal{O}_{E}(-1,0)[2]\right) \cong k
$$

and all the other sets of morphisms between their shifts vanish. Thus $\tilde{C}$ is not equivalent to $C$ or $C_{1}$. However, we can prove that $\tilde{C}$ is indeed not admissible: 
Lemma 4.2. $\tilde{C}$ is not saturated.

Proof. Since $\mathcal{O}_{E}(-1,0)$ is exceptional, it generates a saturated subcategory of $\tilde{C}$. Therefore, it is sufficient to prove that its left orthogonal

$$
\tilde{C}^{\prime}={ }^{\perp}\left\langle\mathcal{O}_{E}(-1,0)\right\rangle \subset \tilde{C}
$$

is not saturated. We decompose the other object $\mathcal{O}_{E}(0,-1)$ by a distinguished triangle

$$
c_{1} \rightarrow \mathcal{O}_{E}(0,-1) \rightarrow \mathcal{O}_{E}(-1,0)[2] \rightarrow c_{1}[1] .
$$

where $c_{1} \in \tilde{C}^{\prime}$. Then $\tilde{C}^{\prime}$ is generated by $c_{1}$, and we have

$$
\operatorname{Hom}\left(c_{1}, c_{1}[p]\right) \cong \begin{cases}k & \text { if } p=0,3 \\ 0 & \text { otherwise. }\end{cases}
$$

Thus $\tilde{C}^{\prime}$ is equivalent to a category generated by an object $\mathcal{O}_{M}$ in the derived category $D^{b}(\operatorname{Coh}(M))$ for a Calabi-Yau 3-fold $M$. Such an object is called a spherical object. Since $D^{b}(\operatorname{Coh}(M))$ is indecomposable and its Serre functor is isomorphic to a shift functor [3], $\left\langle\mathcal{O}_{M}\right\rangle$ is not saturated, hence neither is $\tilde{C}^{\prime}$.

We can also check this fact directly. Let $F:\left(\tilde{C}^{\prime}\right)^{\text {op }} \rightarrow(k$-vect $)$ be a cohomological functor such that $F\left(c_{1}\right) \cong k$ and $F\left(c_{1}[p]\right)=0$ for $p \neq 0$. Then $F$ is not representable by any object $c_{2} \in \tilde{C}^{\prime}$. Indeed, if $F(c) \cong \operatorname{Hom}\left(c, c_{2}\right)$ for arbitrary $c \in \tilde{C}^{\prime}$, then $\sum_{p}(-1)^{p} \operatorname{dim} \operatorname{Hom}\left(c_{1}[p], c_{2}\right)$ should be even, a contradiction.

\subsection{Case 2}

$E$ is isomorphic to a projective plane $\mathbb{P}^{2}$, and the normal bundle of $E$ is isomorphic to $\mathcal{O}_{E}(-2)$.

Let

$$
C=\left\langle\mathcal{O}_{E}(-1)\right\rangle \subset D^{b}(\operatorname{Coh}(Y)) .
$$

Since $\mathcal{O}_{E}(-1)$ is an exceptional object, $C$ is an admissible subcategory. We note that $\left(\mathcal{O}_{E}(-2), \mathcal{O}_{E}(-1)\right)$ is not an exceptional collection as in Case 1. Indeed, we have

$$
\operatorname{Hom}\left(\mathcal{O}_{E}(-1), \mathcal{O}_{E}(-2)[3]\right) \cong \operatorname{Hom}\left(\mathcal{O}_{E}(-2), \mathcal{O}_{E}(-2)\right)^{*} \neq 0 .
$$


Thus these two objects may not generate an admissible subcategory.

Let $\mathcal{X}$ be the smooth Deligne-Mumford stack associated to the variety $X$ which has only a quotient singularity $P$, and let $\pi: \mathcal{X} \rightarrow X$ be the projection. We know that $D \cong D^{b}(\operatorname{Coh}(\mathcal{X}))$ by [9], and the latter is the correct category. We shall identify these categories in the following.

We have $f_{*} \mathcal{O}_{P}(1)=0$. But $\mathcal{O}_{P}(1)$ is not an exceptional object, because $\operatorname{Hom}^{2}\left(\mathcal{O}_{P}(1), \mathcal{O}_{P}(1)\right) \neq 0$. We know also that $S_{D}^{2}(d) \cong d[6]$ if $d \in D$ and $f_{*} d=0$, but

$$
S_{D}\left(\mathcal{O}_{P}(1)\right) \cong \mathcal{O}_{P}[3] \nRightarrow \mathcal{O}_{P}(1)[3]
$$

Lemma 4.3. $D$ is minimal.

Proof. Suppose that there is a semi-orthgonal decomposition $D=\left\langle C^{\prime}, D^{\prime}\right\rangle$ such that $f^{*} \operatorname{Perf}(X) \subset D^{\prime}$. Let $c \in C^{\prime}$ be a non-zero object. Since $f_{*} c=0$, $c$ is supported at the point $P$. Since $\operatorname{Hom}\left(\mathcal{O}_{\mathcal{X}}, c[n]\right)=0$ for any $n, H^{n}(c)$ should be of the form $\mathcal{O}_{P}(1)^{c_{n}}$. Any object $d \in D^{\prime}$ has a finite resolution whose terms are of the form $\mathcal{O}_{\mathcal{X}}^{a_{n}} \oplus \mathcal{O}_{\mathcal{X}}(1)^{b_{n}}$ near $P$. If $b_{n} \neq 0$ for some $n$ and for any choice of such a resolution, then we have $\operatorname{Hom}(d, c[m]) \neq 0$ for some $m$, a contradiction. Therefore, $b_{n}=0$ for all $n$, and $d \in f^{*} \operatorname{Perf}(X)$, a contradiction to Lemma 2.1.

\subsection{Case 3}

$E$ is isomorphic to a singular quadric surface, and the normal bundle of $E$ is isomorphic to $\mathcal{O}_{E}(-1)$.

This case has only a partial answer. Let $l$ be a ruling. Then $\mathcal{O}_{E}(1) \cong$ $\mathcal{O}_{E}(2 l)$. There is an exact sequence

$$
0 \rightarrow \mathcal{O}_{E}(-3 l) \rightarrow \mathcal{O}_{E}(-2 l)^{2} \rightarrow \mathcal{O}_{E}(-l) \rightarrow 0
$$

The Serre functor of $D^{b}(\operatorname{Coh}(Y))$ is given by $\otimes \mathcal{O}_{Y}(E)[3]$, and we have

$$
\operatorname{Hom}\left(\mathcal{O}_{E}(-l), \mathcal{O}_{E}(-l)[2]\right) \cong \operatorname{Hom}\left(\mathcal{O}_{E}(-l), \mathcal{O}_{E}(-3 l)[1]\right)^{*} \neq 0
$$

hence $\mathcal{O}_{E}(-l)$ is not an exceptional object.

Let

$$
C=\left\langle\mathcal{O}_{E}(-1)\right\rangle
$$

and $D={ }^{\perp} C$. Since $\mathcal{O}_{E}(-1)$ is an exceptional object, $C$ is admissible. We have $\mathcal{O}_{E}(-l) \in D$. The above sequence implies that

$$
j ! \mathcal{O}_{E}(-3 l) \cong \mathcal{O}_{E}(-l)[-1]
$$


where $j^{!}$is the right adjoint to $j_{*}: D \rightarrow D^{b}(\operatorname{Coh}(Y))$. Therefore,

$$
S_{D}\left(\mathcal{O}_{E}(-l)\right) \cong \mathcal{O}_{E}(-l)[2]
$$

We expect that if $c \in D$ is right orthogonal to $f^{*} \operatorname{Perf}(X)$, then $S_{D}(c) \cong$ $c[2]$. We note that the shift number 2 is different from usual $3=\operatorname{dim} X$. We can only prove a partial result:

Lemma 4.4. Let $c \in D$ such that $c \in\left(f^{*} \operatorname{Perf}(X)\right)^{\perp}$. Assume in addition that there exists $d \in D^{b}(\operatorname{Coh}(E))$ such that $c=i_{*} d$ for $i: E \rightarrow Y$. Then $S_{D}(c) \cong c[2]$.

Proof. By a generalization of [1], $d$ has a two sided resolution whose terms are direct sums of the sheaves $\mathcal{O}_{E}, \mathcal{O}_{E}(-l)$ and $\mathcal{O}_{E}(-2 l)$. Since

$$
\operatorname{Hom}_{E}\left(\mathcal{O}_{E}, d[n]\right) \cong \operatorname{Hom}_{Y}\left(\mathcal{O}_{Y}, c[n]\right)=0
$$

for any $n$, the terms do not contain $\mathcal{O}_{E}$. On the other hand, since

$$
\operatorname{Hom}_{Y}\left(c, \mathcal{O}_{E}(-2 l)[n]\right)=0
$$

for any $n$, the terms do not contain $\mathcal{O}_{E}(-2 l)$ either. Since $S_{D}\left(\mathcal{O}_{E}(-l)\right) \cong$ $\mathcal{O}_{E}(-l)[2]$, we have our assertion.

Let $B$ be the full subcategory of $D^{b}(\operatorname{Coh}(Y))$ consisting of objects whose supports are contained in $E$. Then $B$ is generated by $\mathcal{O}_{E}$-modules as a triangulated category. But we note that the inclusion functor $D^{b}(\operatorname{Coh}(E)) \rightarrow$ $B$ is not fully faithful because there are more extensions in $B$.

In order to prove the above expectation, one would need more geometric argument. Anyway, if it is true, then the minimality of the category $D$ will follow from its indecomposability.

Lemma 4.5. $D$ is indecomposable.

Proof. Assume that $D=C^{\prime} \oplus D^{\prime}$. Since $\operatorname{Hom}\left(\mathcal{O}_{Y}, \mathcal{O}_{Y}\right) \cong k, \mathcal{O}_{Y} \in D$ is indecomposable. We may assume that $\mathcal{O}_{Y} \in D^{\prime}$.

Let $x \in E$ be an arbitrary point, and let $d=j ! \mathcal{O}_{x}$ for the right adjoint functor $j^{!}$of $j: D \rightarrow A=D^{b}(\operatorname{Coh}(Y))$. We have a distinguished triangle

$$
d \rightarrow \mathcal{O}_{x} \rightarrow \operatorname{Hom}^{\bullet}\left(\mathcal{O}_{x}, \mathcal{O}_{E}(-1)\right)^{*} \otimes \mathcal{O}_{E}(-1) \rightarrow d[1]
$$


The third term is isomorphic to $\mathcal{O}_{E}(-1)[2] \oplus \mathcal{O}_{E}(-1)[3]$. Then

$$
\operatorname{Hom}(d, d) \cong \operatorname{Hom}\left(d, \mathcal{O}_{x}\right) \cong \operatorname{Hom}\left(\mathcal{O}_{x}, \mathcal{O}_{x}\right) \cong k
$$

because

$$
\operatorname{Hom}\left(\mathcal{O}_{E}(-1)[k], \mathcal{O}_{x}\right)=0
$$

for $k>0$. Therefore, $d$ is indecomposable. Since

$$
\operatorname{Hom}\left(\mathcal{O}_{Y}, d\right) \cong \operatorname{Hom}\left(\mathcal{O}_{Y}, \mathcal{O}_{x}\right) \neq 0
$$

we have $d \in D^{\prime}$.

Let $c \in C^{\prime}$ be an arbitrary object such that $c \neq 0$. Then the support of $c$ is contained in $E$. Thus there exists $x$ and $p$ such that

$$
\operatorname{Hom}(c, d[p]) \cong \operatorname{Hom}\left(c, \mathcal{O}_{x}[p]\right) \neq 0
$$

a contradiction.

\section{Questions}

Based on the above observation, we would like to ask the following questions:

(1) Let $X$ be a projective variety with only terminal singularities, and $f: Y \rightarrow X$ a resolution of singularities. Does there exist a minimal saturated subcategory of $D^{b}(\operatorname{Coh}(Y))$ which contains $f^{*} \operatorname{Perf}(X)$ ? Are two such minimal saturated subcategories equivalent?

(2) More generally, let $X$ be a smooth projective variety. Does the category $D^{b}(\operatorname{Coh}(X))$ have finite length with respect to semi-orthogonal decompositions? Does a Jordan-Hölder type theorem hold for semi-orthogonal decompositions of saturated categories?

(3) It would be nice if we have more method for testing the admissibility of a subcategory in general situation (cf. Lemma 4.2).

(4) Can a geometric argument be given to establish the expectation from Subsection 4.3? (added by a referee)

\section{References}

[1] A. A. Beilinson. Coherent sheaves on $\mathbb{P}^{n}$ and problems of linear algebra. Funct. Anal. Appl. 12(1978), 214-216. 
[2] A. I. Bondal and M. M. Kapranov. Representable functors, Serre functors, and reconstructions. Izv. Akad. Nauk SSSR Ser. Mat. 53 (1989), no. 6, 1183-1205; translation in Math. USSR-Izv. 35 (1990), no. 3, $519-541$.

[3] A. I. Bondal and D. O. Orlov. Semiorthogonal decomposition for algebraic varieties. alg-geom/9506012.

[4] Alexei Bondal and Michel Van den Bergh. Generators and representability of functors in commutative and noncommutative geometry. math.AG/0204218. Mosc. Math. J. 3 (2003), no. 1, 1-36, 258.

[5] Tom Bridgeland. Equivalences of triangulated categories and FourierMukai transforms. math.AG/9809114. Bull. London Math. Soc. 31(1999), 25-34.

[6] Sergei I. Gelfand and Yuri I. Manin. Methods of homological algebra. Second edition. Springer Monographs in Mathematics. Springer-Verlag, Berlin, 2003. $\mathrm{xx}+372 \mathrm{pp}$.

[7] Yujiro Kawamata. Francia's flip and derived categories. math.AG/0111041. in Algebraic Geometry (a volume in Memory of Paolo Francia), Walter de Gruyter, 2002, 197-215.

[8] Yujiro Kawamata. Log Crepant Birational Maps and Derived Categories. math.AG/0311139. J. Math. Sci. Univ. Tokyo 12 (2005), 211231.

[9] Yujiro Kawamata. Derived Categories of Toric Varieties. math.AG/0503102. Michigan Math. J. 54 (2006), 517-535.

[10] Yujiro Kawamata, Katsumi Matsuda and Kenji Matsuki. Introduction to the minimal model problem. in Algebraic Geometry Sendai 1985, Advanced Studies in Pure Math. 10 (1987), Kinokuniya and North-Holland, 283-360. http://faculty.ms.u-tokyo.ac.jp/ kawamata/index.html

[11] Alexander Kuznetsov. Derived categories of cubic and $V_{14}$ threefolds. math.AG/0303037. 
[12] Amnon Neeman. The Grothendieck duality theorem via Bousfield's techniques and Brown representability. alg-geom/9412022. J. Amer. Math. Soc. 9 (1996), no. 1, 205-236.

[13] Dmitri Orlov. Projective bundles, monoidal transformations, and derived categories of coherent sheaves. Russian Acad. Sci. Izv. Math. 41(1993), 133-141.

[14] Dmitri Orlov. Derived categories of coherent sheaves and motives. math.AG/0512620.

Department of Mathematical Sciences, University of Tokyo, Komaba, Meguro, Tokyo, 153-8914, Japan

kawamata@ms.u-tokyo.ac.jp 